\title{
Biodiversité, une éthique socio-environnementale
}

\section{Francis Gaillard}

\section{(2) OpenEdition \\ Journals}

Édition électronique

URL : http://journals.openedition.org/ere/2016

DOI : 10.4000/ere.2016

ISSN : 2561-2271

Éditeur

Centr'ERE

\section{Référence électronique}

Francis Gaillard, «Biodiversité, une éthique socio-environnementale», Éducation relative à

l'environnement [En ligne], Volume 8 | 2009, mis en ligne le 20 décembre 2009, consulté le 21 février 2020. URL : http://journals.openedition.org/ere/2016 ; DOI : 10.4000/ere.2016 


\title{
Biodiversité, une éthique socio- environnementale
}

\author{
Francis Gaillard
}

1 Parmi les grands bouleversements que la crise environnementale suscite, l'attention portée à la biodiversité revêt un intérêt particulier. En effet, c'est la question de la place que l'être humain laisse aux autres espèces qui est posée, et finalement celle de la sienne propre dans les écosystèmes.

2 Question éminemment éthique, qui entre en ligne de compte, le plus souvent de façon sous-jacente, au moment du choix des politiques environnementales et des actions qui en découlent. Le concept de biodiversité dépasse donc le cadre strict des sciences biologiques qui l'ont créé et affecte la vie sociale.

3 Les débats éthiques ont mis à jour diverses approches de la nature : anthropocentrée, biocentrée ou écocentrée; des valeurs éthiques, parfois contradictoires, qui ont souvent fonctionné en opposant les humains et les non humains, une vision dichotomique, dualiste, dont il faut sortir pour intégrer la complexité de mondes en interaction, ce qui est le contexte de la gestion de la crise environnementale. Ceci implique des responsabilités individuelles et collectives dans un rapport dialogique avec l'environnement, où les acteurs sociaux expérimentent, négocient, évaluent, réajustent sans cesse leurs actes, vis-à-vis d'eux-mêmes et des non humains. La prise en compte de la biodiversité, comme but et cadre d'action, contribue ainsi à définir une éthique socio-environnementale.

Pour en comprendre les fondements et l'émergence, il est nécessaire de voir quand et comment la biodiversité a commencé à occuper le terrain social. Puis, au travers d'une étude de cas de sauvegarde d'espèce menacée, identifier les leviers actuels de l'action en faveur de la biodiversité d'où émerge la question de l'éthique socioenvironnementale. Enfin, définir les bases de cette éthique, que l'éducation relative à l'environnement pourrait alimenter autant qu'elle s'en nourrirait. 


\section{Intrusion de la biodiversité dans la sphère sociale}

5 Si, à l'origine, en 1988, le terme biodiversité a été inventé et utilisé pour rendre compte d'un forum américain sur la diversité biologique - signifiant alors la totalité de toutes les variations de tout le vivant - très vite il a été le vecteur de préoccupations politiques et d'enjeux économiques dans le cadre de conventions internationales. La Convention sur la diversité biologique est signée en 1992 au cours de la conférence de Rio - la biodiversité est alors définie comme l'ensemble des espèces, des écosystèmes et des gènes - c'est l'entrée dans la sphère socio-économique: la biodiversité est une ressource appropriable et brevetable. Puis, en 2002, à Johannesburg, la biodiversité s'enrichit de la sociodiversité, c'est-à-dire des usages et des cultures qui ont accompagné l'évolution de cette biodiversité.

6 De concept biologique, la biodiversité est devenue un ensemble de biens marchands susceptibles d'appropriation, puis un cadre pour aborder des problèmes de gouvernance, en réponse à l'inadéquation des modes politiques pour réguler les relations entre les différents acteurs sociaux et les écosystèmes. De fait, les préoccupations liées à la dégradation de l'environnement se sont déplacées vers celles qui sont liées à l'organisation des sociétés humaines (Aubertin, 2002).

7 La notion de biodiversité est entrée rapidement dans le champ de la politique et des préoccupations du grand public. Cela peut probablement s'expliquer par la gravité de la crise, puisque le taux de disparition des espèces est de 1000 à 10000 fois supérieur au rythme normal d'extinction. S'il n'y a pas actuellement de preuves scientifiques que la diminution de la biodiversité pourrait remettre en cause la vie même sur terre, la communauté scientifique nourrit pourtant d'importantes inquiétudes sur le fait, par exemple, que certaines potentialités soient définitivement perdues, que ce soit en termes de ressources génétiques, de molécules utiles, ou encore de perte d'aménités.

8 Les médias ont relayé largement ces menaces par des émissions grand public, souvent au travers d'exemples exotiques, dans le quotidien des téléspectateurs. Cette fulgurante appropriation de la notion de biodiversité, et ce qu'elle recouvre, montre combien elle touche en profondeur les acteurs sociaux, remettant en cause des certitudes fortement ancrées dans les sociétés modernes : l'humain n'est plus maître et possesseur de la nature.

9 Au niveau politique, en France, la sauvegarde de la biodiversité s'inscrit dans le programme européen Natura 2000. Ce programme consiste à inventorier et sélectionner, sur des bases scientifiques, des espaces de sauvegarde. Ces espaces doivent former à terme une trame dont il faudra assurer par la suite la mise en réseau.

10 L'action politique volontariste, du niveau international jusqu'au niveau local, conjuguée à un fort engouement du grand public, laisserait présager d'un règlement rapide et consensuel du problème. Actuellement, les mesures adoptées couvrent en France $12,4 \%$ du territoire, et, malgré ces efforts, la dégradation de la biodiversité est toujours constatée, au regard du principal indicateur reposant sur le suivi des populations d'oiseaux communs (IFEN, 2007). En outre, dans certains espaces, la mise en place du programme a été très conflictuelle, agriculteurs et chasseurs en étant les principaux pourfendeurs.

11 En fait, la sauvegarde de la biodiversité ne passe pas seulement par des mesures écologiques, réglementaires ou économiques. Elle doit aussi faire l'objet d'une prise en 
compte du contexte social, souvent localisé, et des rapports qui unissent (ou éloignent) ces acteurs sociaux de l'objet de cette sauvegarde.

\section{L'éthique sur le terrain de la biodiversité : sauvetage de l'Outarde}

12 Les actions en faveur de la biodiversité dans le cadre du programme Natura 2000 mettent donc en relation des humains et des non humains dans un objectif de règlement d'une crise, jugée certes préoccupante, mais dont on ne sait pas précisément quelles en seraient les conséquences, et dont les améliorations ne seraient pas immédiatement visibles. Dès lors, la question éthique devient importante, non pas tant pour édicter les valeurs prépondérantes, que pour permettre aux acteurs sociaux de s'entendre sur des finalités et des modes opératoires, mais aussi de faire évoluer leurs propres représentations.

13 Afin d'évaluer les modalités d'implication des acteurs sociaux dans le contexte d'une opération de protection de la biodiversité, une enquête a été menée sur le territoire d'une zone Natura 2000 à Chizé (Poitou-Charentes/France), où le Centre d'Études biologiques, laboratoire du CNRS, travaille à la protection de l'Outarde, un oiseau de plaine menacé de disparition.

14 Ce premier travail d'enquêtes (Gaillard, 2006) a permis d'identifier des logiques d'action à l'œuvre sur ce territoire, au regard de la protection de cet oiseau, en fonction des modes d'habiter de la population locale. Il s'agissait d'établir des liens entre modes de vie des habitants et protection de l'environnement, et on peut en tirer quelques informations utiles sur les fondements éthiques des mesures actuelles de gestion de la biodiversité.

15 La prédominance du social dans cette action de protection apparaît à divers niveaux : dans le choix d'une espèce qu'il faut protéger, et dans les modes de vie ou de production agricole qui en modifient l'évolution. L'accent mis sur une espèce emblématique comme l'Outarde provient tout d'abord d'un consensus, d'un choix social, probablement marqueur identitaire pour certaines catégories d'acteurs. L'Outarde était une espèce chassée, qui est encore assez présente dans la mémoire des agriculteurs les plus anciens ou à la retraite; les ornithologues, eux, savent que le Poitou-Charentes est une des dernières zones de présence de l'Outarde. Il y a donc un enjeu de sauvetage fort. Par ailleurs, les acteurs locaux construisent des espaces en fonction d'une dominante : habiter, produire, se ressourcer, agir pour la collectivité ; mais ces espaces définis sont assez exclusifs, et les frontières qui les délimitent sont porteuses de conflits d'intérêts. Au bout du compte, il est très difficile pour les acteurs de juger, à partir de ces espaces construits, des qualités environnementales afférentes. L'appréciation qu'ils en ont est basée sur des considérations d'ordre général plutôt que sur l'observation de l'impact de leurs pratiques.

Les logiques d'action qui ont une influence sur l'évolution de la population d'outardes sont liées aux modes de vie et de production, celles repérées s'articulent autour de deux grandes tendances, celles qui intensifient l'utilisation de l'espace et celles qui au contraire contribuent à une moindre pression anthropique. Il y a la logique de l'hyper consommation spatiale, que ce soit pour la production agricole, la construction de pavillons et de routes et la logique de l'évitement qui ont une influence négative et 
massive sur la population d'outardes; puis les logiques de décélération et d'appropriation collective qui pourraient avoir un effet positif, mais qui sont très minoritaires.

Les logiques d'action identifiées permettent a posteriori, en creux, de faire ressortir les visions anthropocentrée et écocentrée des acteurs locaux et aussi leurs limites. La gestion du territoire, au travers de la prise en compte d'un problème de biodiversité par Natura 2000, passe par des moyens de concertation limitée à certaines catégories d'acteurs et par une certaine forme d'ingénierie écologique. Les débats sur les finalités et les moyens ont bien lieu, mais tournent parfois à la caricature entre écologistes d'un côté et productivistes de l'autre, chacun sachant cependant qu'il a besoin de l'autre. En effet, en proposant une amélioration de la matrice paysagère, en développant par exemple la culture de la luzerne, favorable aux nichées, avec quelques agriculteurs, il est possible de stabiliser la population d'outardes. Mais globalement, les pratiques n'ont pas été majoritairement modifiées. Les approches technico-économique et technicoscientifique ne semblent pas pouvoir assurer durablement la protection de l'environnement, et ce d'autant, qu'il s'opère une régulation par le bas (Salles, 2006, p.161) dans le cas où la gestion environnementale est à la confluence de choix individuels et de politiques publiques.

Pour autant l'exemple étudié n'est pas négatif au regard de l'évolution de la population d'outardes. D'un point de vue utilitariste, les actions qui ont mêlé scientifiques, militants écologistes, agriculteurs et pouvoirs publics sont efficaces, mais il y a une fragilité du dispositif, et ce d'autant plus que la protection est fondée sur des incitations économiques dont les procédures de financement sont remises en cause à chaque alternance politique.

C'est que peut-être les approches moins utilitaristes n'ont pas été suffisamment explorées. En effet, si l'amélioration des qualités environnementales nécessite des bases scientifiques et techniques maîtrisées, elle ne peut s'y résumer. L'étude des relations qu'entretiennent les humains avec ces objets environnementaux, et entre eux au travers de ces objets, est essentielle pour rendre plus probable une amélioration durable de la situation.

L'éthique socio-environnementale devrait se situer dans cet interstice mouvant et ténu qui fait que humains et non humains ont à se partager des espaces et que l'incidence des uns sur les autres représente un enjeu qui les dépasse en partie, mais qui les concerne directement. Les débats éthiques, que suppose une telle approche, portent sur ce qu'est la définition même de l'humain, et on l'a vu de sa place dans l'écosystème et de la place qu'il laisse aux autres êtres vivants. C'est évidemment un peu vertigineux, mais c'est ce qui devrait permettre de dépasser la controverse dualiste, humain contre nature ou nature contre humain.

\section{Conserver, protéger, débat éthique et questionnement social}

21 La protection de l'environnement pose donc le problème de la place de l'humain. L'environnement est-il une ressource pour l'humain ou bien l'humain est-il nuisible à l'environnement? Pour simplifier à l'extrême, deux visions caractérisent les fondements de l'éthique environnementale, basées sur deux systèmes de valeurs: 
biocentrisme et anthropocentrisme. La polémique a fait rage entre les tenants de la Deep Ecology et ceux d'une écologie au service strict des humains. Pour dépasser ce débat, l'écocentrisme, une sorte de compromis entre les deux positions radicales, tente d'intégrer les activités humaines dans l'environnement naturel, une sorte d'humanisme environnemental. Alliance du «je» et du «nous», une éthique de la fragilité, écrit Corcuff (2002, p. 181), qui «relève tout à la fois d'une responsabilité morale individualisée (mes simples actes individuels pèsent déjà dans la balance) et d'une responsabilité politique collective (les choix collectifs ont des effets majeurs) ».

Les questions éthiques sont multiples, et parfois, la visée de protection, son efficacité, ses buts, sont dilués dans des controverses interminables, des débats conceptuels. Fautil préserver la nature, la sauvegarder, la restaurer, voire l'incarcérer? Au bout du compte, on pourrait commencer à douter que de tels dilemmes permettent d'améliorer la situation sur le plan biologique.

23 C'est que finalement le débat, entre les tenants d'une soumission des humains à la nature et ceux de la soumission de la nature aux intérêts humains, comporte, selon Augustin Berque, une aporie :

[...] celle d'admettre simultanément que l'humain transcende en un sens la nature, quand dans un autre, celle-ci le subsume. En effet, attendre de l'espèce humaine un comportement éthique respectant l'intérêt des autres espèces est ipso facto lui conférer un statut à part, puisqu'on ne peut raisonnablement pas reconnaître à celles-ci un devoir réciproque à notre égard. Autrement dit, le champ de l'éthique et celui de l'écologie restent foncièrement hétérogènes. (Berque, 2004)

Et pour résoudre cette aporie, l'auteur propose un "décentrement: celui de la révolution qui, à l'être vers la mort de l'individu moderne, substituera l'être vers la vie de ce qui fonde en réalité notre existence : être humains sur la Terre » (Berque, 2004).

Un état particulier de l'humain que la philosophe américaine Kate Soper (2001) pense comme une contradiction, non pas pour rendre invivable sa condition d'humain, mais plutôt pour l'extirper de ce dualisme afin de repenser le sens de son identité.

Plutôt que de la révérence, nous devons peut-être devenir plus conscients que notre dépendance est inséparable d'un certain détachement vis-à-vis d'elle. Se rapprocher de la nature, en un sens, c'est être plus angoissé par l'impossibilité de nous identifier à elle ou de l'identifier à nous. Mais, à travers cette prise de conscience, nous devenons en même temps susceptibles de modifier notre sens même de l'identité humaine. (Soper, 2001)

Difficile de dire quelle forme va prendre cette identité humaine modifiée. L'identité est évidemment un processus qui met en jeu l'éducation, le milieu familial, et dans une certaine mesure le milieu professionnel. Repenser l'identité humaine nécessite un questionnement social de l'environnement en tenant compte des caractéristiques contemporaines des problèmes écologiques, à savoir: leur caractère planétaire; l'invisibilité des difficultés écologiques contemporaines, qui nécessitent des médiations scientifiques; leur imprévisibilité et leur non maitrise, d'où la difficulté de prévoir, donc de gouverner, d'où l'intérêt du principe de précaution ; puis leur inertie : puisqu'il y a un décalage entre action et résultats (Bourg, 2003).

La recherche en cours postule que les modalités de la prise en compte de la biodiversité dans la société permettraient de déceler des changements identitaires. Ainsi la biodiversité pourrait agir comme une altérité, une étrangeté intangible, nécessaire, incontournable, dont on ne peut pas faire le tour, ni maîtriser entièrement tous les facteurs, et à partir de laquelle un processus de subjectivation pourrait s'établir, non 
pas pour désenchanter le monde, mais pour en aborder la complexité. À partir de cette distance à l'autre, à soi, on pourrait en effet bâtir à nouveau une identité qui aurait ses traductions dans les altérations de nos positionnements vis-à-vis d'elle dans notre vie quotidienne (Cingolani, 2004).

L'éthique socio-environnementale, c'est donc ce qui pourrait être cette aptitude à modifier nos positionnements vis-à-vis d'une identité qui se construit dans le réajustement incessant de la distance à la nature, à soi et aux autres ; ou encore, ce qui permettrait de définir la nature dans laquelle vivre serait possible.

Contrairement à ce que l'on présente le plus souvent, les problèmes écologiques, ou de protection de la nature ne mettent pas en jeu le conflit entre l'Homme et la nature (l'Homme contre la baleine, le loup ou l'ours...) mais posent la question de savoir dans quelle nature nous voulons vivre. Le choix n'est pas entre l'Homme ou la nature, mais entre un monde uniforme, modelé aux seuls intérêts économiques et un monde divers, laissant place à la pluralité des aspirations humaines, des façons de faire et des manières d'être comme à la pluralité des vivants. (Larrère, 2006)

En prenant en compte la biodiversité, les humains opèrent finalement une métamorphose. Que restera-t-il de cette métamorphose une fois que nous l'aurons poussée à son terme? Quelle sera alors notre singularité ? Quelles voies exploreronsnous pour redéfinir notre propre autonomie et distinction? De quoi aurons-nous besoin pour accepter de vivre aux côtés d'autres espèces? Pour accepter la pluralité des aspirations humaines? Les problèmes que pose ce déplacement opéré par nous, presque à notre insu, sont d'ordre moral, éthique. L'éthique comme spécificité humaine et comme ce sur quoi un changement, une éducation peuvent s'appuyer.

$\mathrm{Au}$ bout du compte, on peut dire que la prise en compte de la biodiversité, et l'éducation qui la porterait, doivent s'articuler pour ne pas rendre impossibles les recompositions identitaires, dans lesquelles la biodiversité doit fonctionner comme une altérité, à la fois proche et irrémédiablement distante. Ainsi, l'éducation relative à l'environnement doit fournir, en parallèle des connaissances écologiques et biologiques, les savoirs sur les conditions d'élaboration et de réception de ces données dans la société, ainsi que les questionnements qu'elles induisent, et ce tant au niveau le plus proche de l'apprenant, dans son environnement immédiat, que dans le cadre plus large de l'humanité.

31 Ainsi, pour en revenir à l'étude de cas sur la zone Natura 2000 et sur l'Outarde, les équipes du Centre d'Études biologiques de Chizé ont ressenti le besoin d'élargir la communauté des humains soucieux de l'Outarde. C'est pourquoi une importante opération de sensibilisation des écoliers de la zone a été conduite autour de la construction et de la pose de nichoirs. Pour aller plus loin, il faudra aussi prendre conscience que protéger l'Outarde c'est lui faire de la place dans notre vie quotidienne, que, concrètement, préserver un écosystème, c'est être à même de penser les modalités de production agricole aussi bien que celles de nos déplacements quotidiens. C'est aussi dépasser la satisfaction immédiate de besoins éphémères au profit de ceux, plus globaux, du collectif humain. C'est accepter enfin que l'évolution de nos comportements ne soit pas qu'un simple artifice, mais bien un nécessaire réajustement identitaire.

32 C'est dans ce contexte que l'éducation relative à l'environnement peut situer le débat éthique. L'objectif n'est pas de savoir qui des humains (ni quelle catégorie des humains) ou des non-humains a le plus de légitimité pour dicter les règles de vie commune dans les écosystèmes, mais d'accepter que la prise en compte de l'altérité environnementale 
et sociale engendre la relativité des priorités et la plasticité des identités qui se construisent dans un rapport dialogique. Une forme d'éthique socio-environnementale.

\section{BIBLIOGRAPHIE}

Aubertin, C. (2002). De Rio à Johannesburg, les avatars de la biodiversité. In Martin J.-Y. (dir.), Développement durable ? Doctrines, pratiques, évaluations (p. 95-123). Paris : IRD Éditions.

Berque, A. (2004). Ce qui fonde l'éthique environnementale. Diogène, 3(207), 3-14.

Bourg, D. (2003). Le nouvel âge de l'écologie. Paris : Descartes et Cie.

Cingolani P. (2004). L'étranger comme catégorie d'action et d'expérience. Socio-Anthropologie, 14. Consulté en mai 2009 sur : http://socio-anthropologie.revues.org/index377.html. Corcuff, P. (2002). La société de verre, Pour une éthique de la fragilité. Paris : Armand Colin.

Gaillard, F. (2006). Outardes et humains en plaine de Niort Sud-Est : un anthroposystème à l'épreuve de la durabilité. Mémoire inédit de M2, Université de Pau et des Pays de l'Adour. IFEN - Institut français de l'environnement. (2007). Les 10 indicateurs clés de l'environnement. Consulté en décembre 2008 sur : http://www.ifen.fr/indicateurs/10-indicateurs-cles-de-l-environnement.html.

Larrère, C. (2006). Éthiques de l'environnement. Multitudes 1(24), 75-84.

Salles, D. (2006). Les défis de l'environnement : démocratie et efficacité. Paris : Syllepse.

Soper, K. (2001). Écologie, nature et responsabilité. Revue du MAUSS semestrielle, 17, 71-93.

\section{AUTEUR}

\section{FRANCIS GAILLARD}

Francis Gaillard est doctorant en sociologie et enseignant d'éducation socio-culturelle au Lycée Agricole Jacques Bujault à Melle (79). Le travail de recherche en sociologie dont il fait état ici s'intéresse à la place de la prise en compte de la biodiversité dans la société. Il est mené dans le cadre d'un travail de doctorant au laboratoire Société Environnement Territoire à Pau (UMR 5603), mais aussi sur un travail d'enquêtes menées avec le Centre d'Études biologiques de Chizé (UPR 1934) auprès de la population locale dans une zone d'étude Natura 2000. 